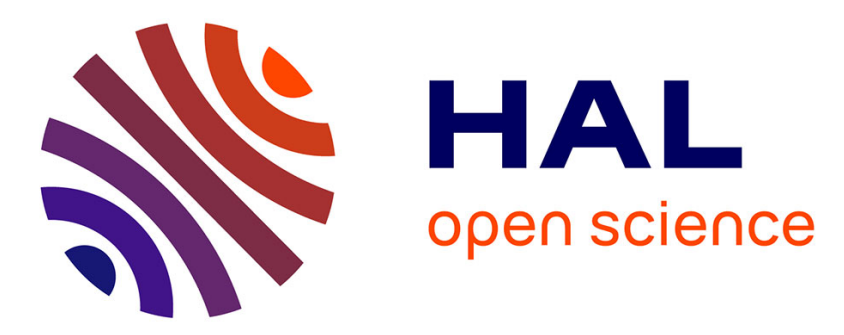

\title{
CARACTÉRISATION ACOUSTIQUE DE MATÉRIAUX TRÈS ABSORBANTS PAR IMPÉDANCEMÉTRIE ÉLECTRIQUE
}

\author{
J. Pouliquen, A. Essaidi
}

\section{- To cite this version:}

J. Pouliquen, A. Essaidi. CARACTÉRISATION ACOUSTIQUE DE MATÉRIAUX TRÈS ABSORBANTS PAR IMPÉDANCEMÉTRIE ÉLECTRIQUE. Journal de Physique IV Proceedings, 1992, 02 (C1), pp.C1-849-C1-852. 10.1051/jp4:19921185 • jpa-00251147

HAL Id: jpa-00251147 https://hal.science/jpa-00251147

Submitted on 1 Jan 1992

HAL is a multi-disciplinary open access archive for the deposit and dissemination of scientific research documents, whether they are published or not. The documents may come from teaching and research institutions in France or abroad, or from public or private research centers.
L'archive ouverte pluridisciplinaire HAL, est destinée au dépôt et à la diffusion de documents scientifiques de niveau recherche, publiés ou non, émanant des établissements d'enseignement et de recherche français ou étrangers, des laboratoires publics ou privés. 


\section{CARACTÉRISATION ACOUSTIQUE DE MATÉRIAUX TRÈS ABSORBANTS PAR IMPÉDANCEMÉTRIE ÉLECTRIQUE}

\section{J. POULIQUEN et A. ESSAIDI}

Faculté Libre des Sciences de Lille, Laboratoire d'Acoustique-Ultrasons (UA 253), 13 rue de Toul, F-59046 Lille cedex, France

We present a method to characterize acoustically a medium having a large absorption $\left(\beta<200 \mathrm{~m}^{-1}\right)$ using only electrical impedance or admittance measurement in C.W. A simulation is given in order to determine the restoration of the characteristics. An example of measurement is proposed and the influence of measurement imprecision on the results is also studied.

L'intérêt de caractériser des matériaux acoustiques par impédancemétrie électrique est multiple: pouvoir utiliser des matériels de mesure électrique conventionnels, nécessiter des échantillons peu épais et réclamer peu de compétences acoustiques de l'opérateur. Par ailleurs, le fait d'utiliser des échantillons minces minimise les effets de diffraction qui peuvent devenir importants en basse fréquence vu les dimensions habituellement limitées des transducteurs.

Des études antérieures [ 1 à 3 ] étaient consacrées à la mesure de la célérité $C_{1}$ des ondes de compression et du coefficient d'absorption $\beta$ dans les matériaux isotropes avec $\beta<30 \mathrm{~m}^{-1}$ à des fréquences voisines ou supérieures à $1 \mathrm{MHz}$. Dans ce cas, l'impédance acoustique caractéristique de la ligne $Z_{0}=\rho_{0} C_{1} S$ ( $\rho_{0}$, masse volumique de l'échantillon, S section du champ acoustique ) est réelle.

En toute rigueur cette impédance acoustique s'exprime par

$$
Z_{0}{ }^{\prime}=Z_{0}(1-j \alpha)[4] \text { avec } \alpha=\beta C_{1} /(2 \pi f) .\left(^{*}\right)
$$

Si $\beta=30 \mathrm{~m}^{-1}, \mathrm{C}_{\mathrm{l}}=1000 \mathrm{~m} / \mathrm{s}, \mathrm{f}=1 \mathrm{MHz}, \alpha \approx 0,005$, $\alpha$ peut être négligé devant 1 . Par contre si $\beta$ augmente et $\mathrm{f}$ diminue cette approximation n'est plus valable $\left(\beta=100 \mathrm{~m}^{-1}, \mathrm{C}_{\mathrm{l}}=1000 \mathrm{~m} / \mathrm{s}, \mathrm{f}=50 \mathrm{kHz}, \alpha \approx 0,3\right)$. On se place délibérément dans ce cas.

\section{Détermination de $C_{l}$ et $\beta$ à partir de l'impédance acoustique de la ligne.}

L'impédance acoustique d'une ligne avec perte est donnée par $Z=Z_{0}(1-j \alpha)$ th $\gamma \mathbf{L}$ avec $\gamma=\beta+j q, q=2 \pi f / C_{1}$, nombre d'onde. Comme th $\gamma L=(\operatorname{sh} 2 \beta L-j \sin 2 q L) / D \quad$ (2) avec $D=\operatorname{ch} 2 \beta L-\cos 2 q L$, si $L$ désigne la longueur de la ligne acoustique réalisée dans le matériau à caractériser.

L'expression (1) devient

$$
Z=Z_{0}\left(\frac{A+B \alpha}{D}\right)+j\left(\frac{B-A \alpha}{D}\right)
$$

(*) Les lettres en caractères gras désignent des grandeurs complexes

$Z, Y$, lettres capitales des grandeurs acoustiques

$z, y$, lettres minuscules des grandeurs électriques 
branche motionnelle $z=Z / 4 N^{2}$ ou (re) $\left.+j(\mathrm{im})=\left(R_{e}+j \delta_{m}\right) / 4 \mathrm{~N}^{2}\right)$ (4). A toute fréquence on a la relation $y_{\mathbf{a b}}=j \omega \mathrm{C}_{\mathbf{o}}+\mathbf{y}_{22^{\prime}}(5)\left(\mathbf{y}_{22}\right.$ admittance de la branche motionnelle ). Mesurant $\mathbf{y}_{\mathbf{a b}}$ et connaissant $\mathrm{C}_{\mathbf{0}}$, l'admittance $\mathbf{y}_{22}$ peut être calculée, pour le transducteur seul $\left(\mathrm{y}_{22} \mathbf{T}_{2}\right)$ et le transducteur chargé $\left(\mathrm{y}_{22} \mathrm{C}_{22^{\prime}}\right)$. Par différence on obtient

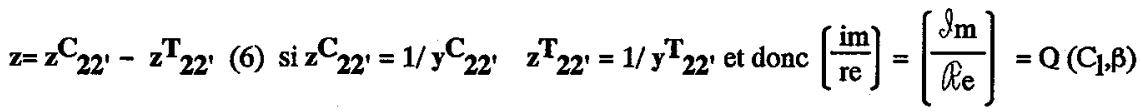

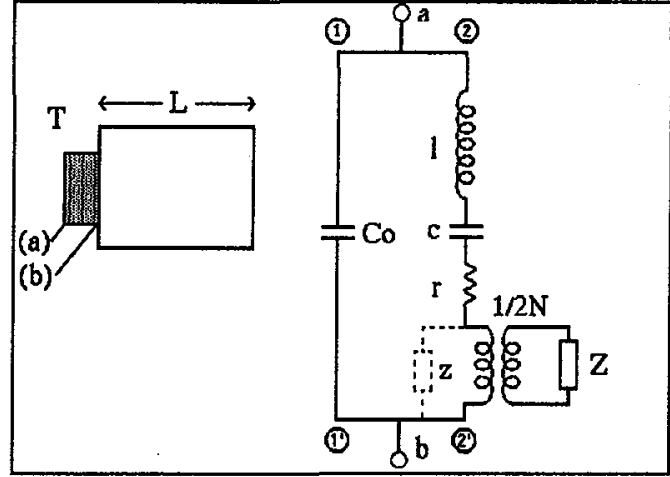

Fig $1:$ Ligne acoustique

Fig2 : Schéma équivalent Pour obtenir $C_{1}$ et $\beta$ il suffit donc de mesurer $C_{0}, y_{a b}$ transducteur seul puis chargé par l'échantillon. La valeur de $C_{0}$ est donnée par la relation $\mathrm{C}_{\mathrm{o}}=\mathrm{C}_{\mathrm{BF}}\left[\frac{\mathrm{fo}}{\mathrm{f} \infty}\right]^{2} \quad$ (7) où $\mathrm{C}_{\mathrm{BF}}$ désigne la capacité mesurée en basse fréquence et $f_{0}, f_{\infty}$ respectivement les fréquences de résonance et d'antirésonance du transducteur seul.

\section{Remarques}

La seule caractéristique du transducteur à connaître est $C_{o}$. Elle peut être mesurée une fois pour toute, mais dépend légèrement de la température.

L'influence du liant a, ici, été négligée. En effet, l'impédance électrique vue au travers de la couche de liant a pour valeur $\quad z=z_{0} \frac{\text { th2 } 2 \gamma L+\left(z_{c} / z_{0}\right) \text { tg qe }}{1-\left(z_{c} / z_{0}\right)^{-1} \text { tg qe th } 2 \gamma L}$

$z_{c}$ impédance acoustique du liant.

Si tg qe $=\operatorname{tg} \frac{2 \pi f e}{v_{c}} \rightarrow 0$ l'impédance se réduit à $\quad z=z_{0}$ th $2 \gamma L$

Pour $\mathrm{e}=5 \mu \mathrm{m}, \mathrm{f}=200 \mathrm{kHz}, \mathrm{v}_{\mathrm{c}}=1000 \mathrm{~m} / \mathrm{s} \quad \operatorname{tg} \mathrm{ge}=0,006$

\section{Mesures}

On a exploité cette méthode pour une lame de P.V.C. transparent ayant $4 \mathrm{~mm}$ d'épaisseur. Le transducteur utilisé ( $\phi=45 \mathrm{~mm} ; \mathrm{e}=10 \mathrm{~mm}$ ) présente les diagrammes d'admittance suivants, selon qu'il est seul (figure 3 ) ou chargé ( figure 4 ).

$$
\mathrm{C}_{\mathrm{BF}}=1,001 \mathrm{nF} \pm 0,001 \mathrm{nF} \quad \mathrm{f}_{\mathrm{o}}=268,473 \mathrm{~Hz} \pm 5 \mathrm{~Hz} \quad \mathrm{f}_{\infty}=295,680 \mathrm{~Hz} \pm 500 \mathrm{~Hz} .
$$

La relation (7) donne $\mathrm{C}_{\mathrm{O}}=0,824 \mathrm{nF} \pm 0,001 \mathrm{nF}$.

Toutes les mesures faites à $22^{\circ} \mathrm{C} \pm 0,25^{\circ} \mathrm{C}$ sont récapitulées au tableau 2 .

Tableau 2 - Mesures

\begin{tabular}{|c|c|c|c|c|c|}
\hline & & \\
\hline & Transducteur & seul & Transducteur & chargé & \\
\hline$f(\mathrm{kHz})$ & $\operatorname{Re}(\mathrm{mS})$ & lm (mS) & $\operatorname{Re}(\mathrm{mS})$ & $d \mathrm{~m}(\mathrm{mS})$ & $\bar{Q}$ \\
\hline 267,270 & $5,350 \pm 0,001$ & $20,110 \pm 0,001$ & $6,589 \pm 0,001$ & $-0,388 \pm 0,001$ & 0,6868 \\
\hline 268,680 & $38,710 \pm 0,001$ & $-38,260 \pm 0,001$ & $4,915 \pm 0,001$ & $-2,092 \pm 0,001$ & 0,6764 \\
\hline
\end{tabular}

La résolution de l'équation (3) pour chacune des fréquences donne comme couple commun $\beta=114 \pm 0 \mathrm{~m}^{-1}$ et $\mathrm{C}_{1}=1620 \pm 0 \mathrm{~m} / \mathrm{s}$. La figure 4 illustre ce résultat et montre l'unicité de solution.

En choisissant d'autres fréquences de mesure pour des points pas trop voisins de l'axe des ordonnées, on obtient des valeurs voisines de $\beta$ et $\bar{C}_{1}$. Voici les valeurs moyennes pour quatre couples de points :

$$
\begin{array}{ll}
\beta=114 \mathrm{~m}^{-1} \pm 2,3 \mathrm{~m}^{-1} & \mathrm{C}_{\mathrm{l}}=1627 \mathrm{~m} / \mathrm{s} \pm 16,5 \mathrm{~m} / \mathrm{s} \\
\text { soit } \Delta \beta / \beta<2 \% & \Delta \mathrm{C}_{\mathrm{l}} / \mathrm{C}_{1}<1 \%
\end{array}
$$


en posant : $A=\operatorname{sh} 2 \beta L$ et $B=\sin 2 q L$. L'équation (1') permet d'écrire

$$
\operatorname{lm}(\mathbf{Z}) / \mathscr{Q}(\mathbf{Z})=\frac{\mathrm{B}-\mathrm{A} \dot{\alpha}}{\mathrm{A}+\mathrm{B} \alpha}=Q\left(\mathrm{C}_{1}, \beta\right)
$$

quantité qui ne dépend plus de $Z_{0} \cdot \operatorname{lm}(Z)$ et $\mathbb{R}(Z)$ désignent respectivement les parties réelle et imaginaire de (Z).

Pour des échantillons de faible épaisseur la mesure à 2 fréquences $f_{1}$ et $f_{2}$ de $Q\left(C_{1}, \beta\right)$ fournit 2 équations non linéaires dont la solution unique donne $C_{1}$ et $\beta$. Pour des valeurs de $A$ élevées ( $\beta$ ou $L$ grand ) $A \gg B$ et $Q \sim \alpha=-\frac{\beta C_{l}}{4 \pi f}$, ce type de solution devient impossible. Dans ce cas il faut connaître $C_{1}$ pour calculer $\beta$ ou inversement et une seule mesure suffit.

L'algorithme qui résout (3) est simple : pour chaque valeur plausible de $C_{1}$ on calcule $\beta$ qui satisfait l'équation. Parmi les couples $\left(C_{1}, \beta\right)$ correspondant aux fréquences $f_{1}$ et $f_{2}$, les deux plus voisins donnent les valeurs cherchées de $\mathrm{C}_{1}$ et $\beta$.

\section{Remarque}

Si $\alpha$ peut être négligé, $Q\left(C_{1}, \beta\right)=\frac{\operatorname{sh} 2 \beta L}{\sin 2 q L}$ et $\beta=\frac{1}{2 L} \operatorname{argsh}\left\{Q \sin \left[\frac{4 \pi f L}{C_{l}}\right]\right\}$

La méthode reste identique mais l'équation (3) peut être remplacée par (3') plus aisée à résoudre.

\section{Simulation}

A partir de (3) on calcule $Q_{1}$ et $Q_{2}$ correspondant à une ligne définie par les quantités $C_{1}, \beta$ et $L$. Puis à partir de la méthode qui vient d'être exposée on essaie de retrouver $C_{l}$ et $\beta, L$ étant une quantité directement mesurable.

La simulation a été faite pour $\mathrm{f}_{1}=45 \mathrm{kHz}, \mathrm{f}_{2}=55 \mathrm{kHz}, \mathrm{C}_{\mathrm{l}}=2000 \mathrm{~m} / \mathrm{s}, \beta=30,50,90,200,500 \mathrm{~m}^{-1}$ et $\mathrm{L}=0,5 ; 1 ; 2 \mathrm{~cm}$. L'erreur relative de la restitution $\Delta \beta / \beta$ est donnée au tableau 1 selon que l'on introduit les valeurs exactes (Ve) de $Q$ ou les valeurs approchées $(\mathrm{Va})$, valeurs précédentes exprimées avec 3 chiffres significatifs ( sensibilité de lecture d'une mesure ).

Tableau 1 - Simulation

\begin{tabular}{|c|c|c|c||c|c|c|}
\cline { 2 - 7 } \multicolumn{1}{c|}{} & \multicolumn{9}{c|}{ Ve } & \multicolumn{3}{c|}{ Va } & \multicolumn{1}{c|}{ Tableau 1 - Simulation } \\
\hline$\beta \mathrm{m}^{-1}$ & $\mathrm{~L}=0,5 \mathrm{~cm}$ & $1 \mathrm{~cm}$ & $2 \mathrm{~cm}$ & $0,5 \mathrm{~cm}$ & $1 \mathrm{~cm}$ & $2 \mathrm{~cm}$ \\
\hline 30 & 0 & $210^{-5}$ & 0 & $310^{-4}$ & $3,510^{-4}$ & $710^{-4}$ \\
\hline 50 & 0 & $210^{-4}$ & 0 & $1,510^{-4}$ & $2,510^{-3}$ & $\mathrm{X}$ \\
\hline 90 & $1,510^{-4}$ & 0 & $210^{-3}$ & $6,510^{-3}$ & $1,510^{-3}$ & $1,510^{-4}$ \\
\hline 200 & $2,510^{-4}$ & $410^{-5}$ & $\mathrm{X}$ & $210^{-4}$ & $410^{-3}$ & $\mathrm{X}$ \\
\hline 500 & $10^{-5}$ & $\mathrm{X}$ & $\mathrm{X}$ & $\mathrm{X}$ & $\mathrm{X}$ & $\mathrm{X}$ \\
\hline
\end{tabular}

Ceci montre la qualité de la restitution de $\beta$ et la nécessité de se limiter à des épaisseurs faibles. La vitesse exacte est rigoureusement retrouvée car le balayage des vitesses réalisé par l'algorithme contient ces valeurs.

En général, le problème n'a plus de solution si $\beta$ est trop grand. Ce fait commence à $\beta>200$. Pour $\beta=500$ il a été possible de résoudre le problème avec $\mathrm{e}=0,5 \mathrm{~cm}$, mais seulement avec les valeurs exactes; les mesures dans cette région seront probablement inexploitables.

\section{Méthode de mesure de $Q\left(C_{1}, \beta\right)$.}

Un transducteur [T] est mis en contact par un liant convenable avec la ligne acoustique constituée du matériau à caractériser ( fig 1 ). Par application d'une tension électrique entre ses bornes a et b il y excite une onde de compression plane. L'impédance électrique $\mathbf{z}_{\mathbf{a b}}$ ( ou l'admittance $\mathbf{y}_{\mathbf{a b}}=1 / \mathbf{z}_{\mathbf{a b}}$ ) apparaissant entre $\mathbf{a}$ et $\mathbf{b}$ dépend du transducteur et de la ligne acoustique. De la mesure de $z_{\mathbf{a b}}$ ( ou $\mathbf{y}_{\mathbf{a b}}$ ) on se propose de déterminer l'impédance acoustique $Z$ de la ligne, ou du moins la valeur de $Q\left(C_{1}, \beta\right)$. Prés de la résonance le schéma équivalent de l'ensemble correspond à celui de la figure $2\left(\mathrm{C}_{\mathrm{o}}, \mathrm{l}, \mathrm{c}, \mathrm{r}, \mathrm{N}\right.$ caractérisent le transducteur et z l'impédance électrique ramenée dans la 


\section{Remarques}

* Des variations de $\pm 1 \%$ sur $C_{0}$ entrainent des variations de $\pm 0,25 \mathrm{~m}-1$ sur $\beta$ et de $\pm 1 \mathrm{~m} / \mathrm{s}$ sur la célérité.

* Des variations de $\pm 0,005$ sur $\operatorname{Re}(Z)$ et $\operatorname{lm}(Z)$ transducteur seul et transducteur chargé causent des variations de $\pm 1,25 \mathrm{~m}^{-1}$ sur $\beta$ et de $\pm 6 \mathrm{~m} / \mathrm{s}$ sur $C_{1}$.
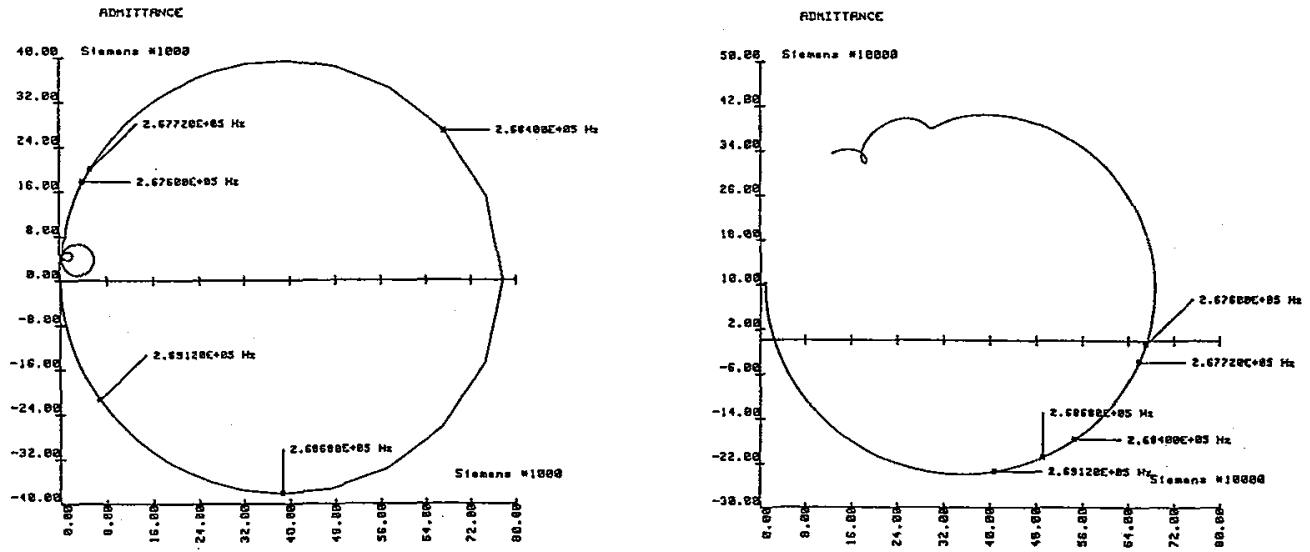

Figure 3 - Transducteur seul

Figure 4 - Transducteur chargé

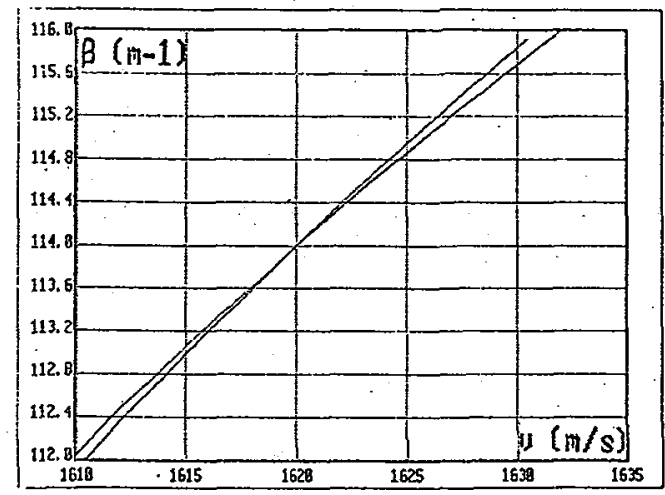

Figure 5 - Unicité de solution

\section{Conclusion}

On a montré la possibilité de caractériser des matériaux acoustiques à faible perte par impédancemétrie électrique $(\beta<200 \mathrm{~m}-1)$.

Une simulation fournit la zone de validité de la méthode ainsi que la précision de restitution des caractéristiques ( célérité et atténuation ) meilleure que $10^{-3}$.

Enfin, une lame de plastique (P.V.C.) de faible épaisseur ( $4 \mathrm{~mm}$ ) a pu être caractérisée. La dispersion des résultats est supérieure aux valeurs de la simulation mais reste inexpliquée par l'imprécision des mesures. L'épaisseur du liant serait peut-être à prendre en considération.

[1] POULIQUENJ. Journée de GAPUS UTC de Compiègne 1989

[2] POULIQUENJ. DEFEBVRE A. 13th ICA Vol 4 p. 305-308 Belgrade 1989

[3] POULIQUEN J. DURIEZ J.M. Physical Acoustics 573-579 Symp. on Phys. Acoust. Courtrai 1990

[4] MERCIER J. Acoustique P.U.F. p.78-79 